\title{
Epithelial-to-endothelial transition and cancer stem cells: two cornerstones of vasculogenic mimicry in malignant tumors
}

\author{
Baocun Sun ${ }^{1,2,3, *}$, Danfang Zhang ${ }^{1,2, *}$, Nan Zhao ${ }^{1,2}$ and Xiulan Zhao ${ }^{1,2}$ \\ ${ }^{1}$ Department of Pathology, Tianjin Medical University, Tianjin, China \\ 2 Department of Pathology, General Hospital of Tianjin Medical University, Tianjin, China \\ ${ }^{3}$ Department of Pathology, Cancer Hospital of Tianjin Medical University, Tianjin, China \\ * These authors have contributed equally to this work \\ Correspondence to: Baocun Sun, email: sunbaocun@aliyun.com \\ Keywords: vasculogenic mimicry, epithelial-to-endothelial transition, cancer stem cells, hypoxia, LPPCN \\ Received: November 09, $2015 \quad$ Accepted: February 14, $2016 \quad$ Published: March 29, 2016
}

Copyright: Sun et al. This is an open-access article distributed under the terms of the Creative Commons Attribution License (CC-BY), which permits unrestricted use, distribution, and reproduction in any medium, provided the original author and source are credited.

\section{ABSTRACT}

Vasculogenic mimicry (VM) is a functional microcirculation pattern in malignant tumors accompanied by endothelium-dependent vessels and mosaic vessels. VM has been identified in more than 15 solid tumor types and is associated with poor differentiation, late clinical stage and poor prognosis. Classic anti-angiogenic agents do not target endothelium-dependent vessels and are not efficacious against tumors exhibiting VM. Further insight into the molecular signaling that triggers and promotes VM formation could improve anti-angiogenic therapeutics. Recent studies have shown that cancer stem cells (CSCs) and epithelium-to-endothelium transition (EET), a subtype of epithelial-to-mesenchymal transition (EMT), accelerate VM formation by stimulating tumor cell plasticity, remodeling the extracellular matrix (ECM) and connecting VM channels with host blood vessels. VM channel-lining cells originate from CSCs due to expression of EMT inducers such as Twist1, which promote EET and ECM remodeling. Hypoxia and high interstitial fluid pressure in the tumor microenvironment induce a specific type of cell death, linearly patterned programmed cell necrosis (LPPCN), which spatially guides VM and endothelium-dependent vessel networks. This review focuses on the roles of CSCs and EET in VM, and on possible novel anti-angiogenic strategies against alternative tumor vascularization.

\section{INTRODUCTION}

Tumor growth and metastasis depends on the development of the tumor's own vascular network [1]. Avascular tumors larger than approximately $1-2 \mathrm{~mm}^{3}$ cannot receive adequate supplies of oxygen, nutrients and host-derived signaling molecules if tumor and host blood vessels are not connected [1]. However, tumor angiogenesis is complex and tumors cannot depend entirely on host endothelial cells for blood vessel formation; thus several tumor angiogenesis pathways exist, including vasculogenic mimicry (VM) and mosaic vessels [2-3].

VM was first reported by Maniotis et al. in 1999, who described the ability of highly aggressive melanoma cells to dedifferentiate into multiple cellular phenotypes, including those with endothelial-like characteristics that could form vessel-like structures to provide blood supply [2]. VM networks are functional vascular-like structures that carry plasma and red blood cells from host blood vessels [2, 4]. Red blood cells are found in VM channels, with no necrosis or hemorrhage around the channels $[2,5]$. Fluorescent dye and activated carbon particles injected into host blood vessels were later detected in VM channels by laser scanning confocal microscopy and light microscopy [2, 4].VM channels are lined with periodic acid-Schiff (PAS) reagent-positive extracellular matrix (ECM) and tumor cells, and endothelial cells and endothelium markers that were not identified in these structures by light microscopy, transmission electron microscopy or immunohistochemistry $[2,6]$. The PASpositive ECM also stains positive for laminin, collagens 
IV and VI, mucopolysaccharide and heparin sulfate glucoprotein (HSPG) [2].

Since its initial identification in human cutaneous and uveal melanomas, VM has been observedin most malignant tumors including hepatocellular carcinoma (HCC), gastric adenocarcinoma, squamous carcinoma, renal cell carcinoma, gastrointestinal stromal tumors (GISTs), tumors of the breast, ovary and prostate and most sarcomas [7-17]. VM has been widely associated with poor prognosis, large tumor volume, poor histological type, higher clinical stage and increased tumor metastasis rate or recurrence frequency [6, 18-20]. The 5-year survival rate of melanoma patients with VM is nearly $0 \%$ [14]. Our research has associated VM with poor prognosis in mesothelial sarcomas, rhabdomyosarcomas and melanomas. Triple-negative breast cancer (TNBC), the breast cancer subtype with the most pessimistic survival rates, exhibits increased VM as compared to other subtypes [19]. Sun, et al. reported that $88.9 \%$ of VMpositive HCC cases, but only about $50 \%$ of VM-negative cases, were at clinical stages III and IV [10]. Moreover, $72.2 \%$ of VM-positive cases suffered from metastasis or recurrence- significantly more than for VM-negative cases. Conventional anti-angiogenic agents, such as TNP470, angiostatin and endostatin [21], have little effect on VM-positive tumors due to the absence of endothelial cells. Sunitinib, an inhibitor of VEGF receptor tyrosine kinase activity, accelerated recurrence and visceral metastasis of VM-positiveTNBC in an animal model [19].

Previous studies showed VM channel formation to involve plasticity of highly malignant tumor cells [22], ECM remodeling $[3,23]$ and connection of VM channels to host blood vessels [2-4]. VM channels, mosaic blood vessels and endothelial vessels coexist in malignant tumors and can switch morphologies depending on the tumor microenvironment [19]. Increasing evidence indicates that cancer stem cells (CSCs) and epithelialto-mesenchymal transition (EMT) are essential in the formation of tumor vasculature $[8,10,16]$. This review focuses on the mechanisms by which tumor cells differentiate into endothelial-like cells and on possible therapeutic strategies to combat these alternative tumor vascularization mechanisms.

\section{VM AND CANCER STEM CELLS}

\section{VM-initiating cells are of particular interest to investigators}

$\mathrm{VM}$ tissues are PAS+/CD31-/CD34-, and do not respond to Ulex europaeus agglutinin-1 by immunohistochemistry [2]. Cells that line VM channels maintainsome characteristics of the malignant tumor, but also have some endothelial cell functions and phenotypes
[2]. VM apparently mimics embryonic angiogenesis $[3,24]$. Embryo vascular networks originate from mesodermal progenitor cells [25]. Angioblasts in blood islands differentiate in situ into endothelial cells and establish an original vessel network in the process of vasculogenesis [25]. In turn, the vasculogenic structure is remodeled to a mature microcirculation system from which endothelial cells proliferate to form new capillaries, in the process of angiogenesis [25].

Previous studies used microarrays to characterize the molecular signature of VM-positive melanoma tumor cells. Bittner, et al. and Seftor, et al. analyzed differentially expressed genes in highly aggressive versuslessaggressive melanomas and identified multiple phenotypespecific genes expressed in the VM-positive cells [22, 26-27]. Aside from melanoma cell-specific genes, genes particular to endothelial cells, epithelial cells, fibroblasts, hematopoietic cells, kidney cells, neuronal tissue, muscle cells and several precursor cell types were found to be expressed in aggressive melanomas [26]. These results indicated that tumor cells that could form VM might revert to a pluripotent, embryonic-like phenotype or stem cell plasticity. Observations of VM in malignant tumors with bi-directional differentiation, such as synoviosarcoma, rhabdomyosarcoma, malignant mesothelioma and epithelioid sarcoma, confirmed that to form VM, tumors must be able to differentiate pluripotently [14].

We analyzed blood vessel patterns in 169 patients with breast cancer and found that more VM-positive patients were in the TNBC group than that in the nonTNBC group [19]. An analysis of the gene expression profiles of 587 patients with TNBC showed enrichment stem cell-specific markers, including ATP binding cassette subfamily A member $8(\mathrm{ABCA} 8)$, protein $\mathrm{C}$ receptor (PROCR), aldehyde dehydrogenase1 (ALDHA1), period circadian clock 1 (PER1) and ATP binding cassette subfamily B member 1 (ABCB1), as well as markers specific to mesenchymal stem cells, such asbone morphogenetic protein 2 (BMP2), endoglin, integrin subunit alpha V (ITGAV), Thy-1 cell surface antigen (THY1), and vascular cell adhesion molecule 1 (VCAM1) [28].

Recent reports indicate that CSCs directly induce VM formation in malignant tumors. Endothelium-like CSCs exhibit enhanced plasticity and can form blood vessel-like channels.

In human renal cell carcinoma, CSC markers CD133 and CD44 were correlated marginally with VM [16], and in HCC tissues, tumor cells lining VM channels expressed SOX2 and OCT4 [29]. Glioma CSCs enriched in the human glioblastoma cell line U87 formed VM channels in xenografts; they also expressed a neural precursor marker, nestin, and multiple stem cell markers, including CD133, Oct4, Nanog and Notch1 [30]. When cultured in differentiation medium, these cells expressed glial cell marker glial fibrillary acidic protein (GFAP) and neuron 
markers that included neuronal class III beta-tubulin and microtubule associated protein 2 [30].

The TNBC cell line MDA-MB-231 can form VMlike channels in matrigel. Hypoxia induced by $\mathrm{CoCl}_{2}$ in vitro accelerated growth of CD133+ MDA-MB-231 cells, which formed more VM channels when reoxygenated; cells lining the new VM channels were CD133+[19]. Wang R,et al. and Lucia Ricci-Vitiani, et al. found that glioblastoma endothelial cells carry the same genomic alterations as tumor cells, suggesting that many tumor endothelial cells have neoplastic origins[31-32]. They also successfully induced glioblastoma stem-like cells to differentiate into functional endothelial cells [31].

The mechanism of CSC function in VM is not yet clear, although the Notch-Nodal signaling pathway has been shown to induce VM formation. The Notch family is essential to inducing stemness and maintaining cancer cells and tumor angiogenesis. Nodal belongs to the Transforming Growth Factor- $\beta$ (TGF- $\beta$ ) superfamily. It can bind to Cripto- 1 and activate anaplastic lymphoma receptor tyrosine kinase (ALK)-4/5/7 and activin receptor IIB, which promotes tumor invasion and metastasis [33]. The Notch receptor intracellular domain breaks off from activated Notch4, which canbind to recombination signaling binding protein-J and form a cytoplasmic complex. Thiscomplex relocates into the nucleus, binds to the Nodal enhancer element and induces Nodal expression [34]. Upregulation of Nodal by Notch4 promoted VM formation in an aggressive melanoma cell line, MV3 [34].

\section{VM AND EMT}

Tumors that are capable of forming VM include both malignant tumors with bi-directional differentiation and epithelial tumors. The transition from bi-directional differentiation tumor cells to VM-lining cells occurs in only one germ layer. The transition of epithelium-derived tumor cells into cells lining VM channels involves mesoblastic cells and endoderm cells, similar to EMT [10]. In the EMT process, epithelial cells lose many of their epithelial characteristics and instead resemble mesenchymal cells, which requires complex changes in cell architecture and behavior [35]. EMT occurs during embryogenesis, wound healing and fibrosis, and is an important step in metastasis [35]. Many EMT-inducing transcription factors, including Snail, Slug, DNA damageresponsive RNA polymerase-degradation factor (dEF1), septin interacting protein 1 (SIP1), Twist1, forkhead box $\mathrm{C} 2$ (FOXC2) and Goosecoid, are associated with tumor invasion and metastasis [(37-40)]. EMT is characterized by changes in the expressionof cell phenotype-specific molecules in response to EMT inducers. Various epithelium-related proteins are down-regulated, including E-cadherin, plakoglobin, occludin, zonula occludins-1, $\alpha$-catenin and claudins 3/4/7 [36]. Emergence of a mesenchymal-like phenotype is indicated by upregulation of proteins such as vascular endothelial (VE)-cadherin, fibronectin, cadherin-2, vimetin, alpha smooth muscle actin and nuclear catenin-1 [37].

Recently, EMT was shown to induce VM formation. VE-cadherin was one of the first molecules identified as a VM promoter in aggressive melanoma [2]. Aggressive melanomas that lack VE-cadherin are incapable of VM[2]. Expression of the EMT-inducing transcription factor, Twist1, is associated with VM in human HCC $[10,38]$. When up-regulated in human HCC HepG2 cell nuclei, Twist1 can promote VE-cadherin transcription, promoting formation of VM channels. This is accompanied by upregulation of VE-cadherin and vimentin, and downregulation of E-cadherin[10]. Similarly, Twist1 overexpression induces EMT in MCF-7 breast cancer cells, which can then form VM channels in 3D culture medium [19]. Conversely, Twist1 knockdown in MDA-MB-231 breast cancer cells impairs VM [19]. Zinc finger E-box binding homeobox 1 (ZEB1) and Slug promoted VM formation in colorectal carcinoma and $\mathrm{HCC}$, respectively, by inducing EMT [39]. Wnt3a expression reduced E-cadherin levels and increased vimentin and $\beta$-catenin expression in colon cancer. Results of in vitro and in vivo experiments showed that Wnt3a overexpression enhanced invasion and VM. Dickkopf-1, an antagonist of the $\mathrm{Wnt} / \mathrm{\beta}$-catenin signaling pathway, partially reversed expression of EMT-associated proteins and VM in Wnt3a-overexpressing cells [40]. Conversely, Wnt5a suppressed colon cancer invasion by inhibiting EMT and VM [41].

During VM channel formation, epithelium-derived tumor cells differentiateinto specific cell types with some endothelial cell markers, such as VE-cadherin and vimentin. We named this process epithelial-to-endothelial transition (EET) in VM formation. EET promoted by EMT-associated inducers crosses two dermal planes, endoderm and mesoderm, and is regulated by precise mechanisms in the tumor microenvironment. Hypoxia, tumor interstitial fluid pressure (IFP) and ECM remodeling promote this process.

\section{PROMOTERS OF EET}

\section{Hypoxia in the tumor microenvironment}

Localized hypoxia in varying degrees is common as tumors develop, and can induce tumor aggression and metastasis by triggering angiogenesis, EMT, genomic instability, apoptotic disorders and increased CSCs [10, 42-43]. Hypoxia is involved in VM formation through several signaling pathways, and is the most potent factor affecting VM [10, 19]. Decreased oxygen levels inhibit the degradation of hypoxia-inducing factors (HIF), allowing for HIF- $1 \alpha$ or HIF- $2 \alpha$ nuclear localization and subsequent 
binding to the hypoxia-response elements of target genes, such as vascular endothelial growth factor (VEGF), VEGF receptors, EMT inducers and stemness-associated genes [44-46]. Under artificial hypoxic conditions induced by blocking a mouse femoral artery, VM channel formation was accelerated in melanoma grafts[47]. Hypoxia also induced VM formation in HCC, breast cancer, ovarian carcinoma, glioblastoma and Ewing sarcoma, both in vivo and in vitro [9-10, 19, 48-49].

Hypoxia has been linked to EET and CSC generation in VM formation. We found that activation of the EMT factor Twist1 was hypoxia-dependent in HCC [10]. Under hypoxic conditions induced by $\mathrm{CoCl}_{2}$, Twist1 translocates to the nucleus and binds to the VE-cadherin promoter to induce EET and VM channel formation [10]. Similarly, hypoxia-associated Twist1 overexpression upregulates VE-Cadherin in MDA-MB-231 TNBC cells, and induces these cells to generate more CSCs to promote VM channels in matrigel [19]. Notch4 is also activated by hypoxia in VM formation. HIF-1 $\alpha$ can bind to the Notch receptor intracellular domain to stabilize the protein, promoting transcription of Nodal and contributing to VM formation [34].

\section{High interstitial fluid pressure and LPPCN}

High interstitial fluid pressure (IFP) in the tumor microenvironment induces both endothelium-dependent vessel formation and VM. Increased IFP caused by rapid cell proliferation and disorganized perfusion in the tumor microcirculation is a barrier to host endothelial cells entering the tumor, resulting in hypoxia at the tumor center [50]. This hypoxia triggers stem-like tumor cells to form VM channels and connect to endotheliumdependent vessels [19]. Mouse melanoma B16 cells were grafted into microenvironments with varying pressures [51]. Melanomas in hind limb skeletal muscle under high pressures were more aggressive than those in the abdominal cavity at low pressures [51]. High microenvironmental pressures increased MMP-2 and MMP-9 expression in melanoma cells, induced ECM remodeling and VM channel formation and accelerated tumor cell invasion [51]. Although GISTs are typically not highly aggressive, VM was identified in 21 of 84 GIST specimens in which MMP-2 and MMP-9 were highly expressed, but endothelium-dependent vessels were rare [13]. GISTs are thought to originate from Cajal's cells in mesodermal tissue or from multipotent mesenchymal stem cells [52]. High interstitial pressures in GISTs as a result of dense tissue limit growth of endothelium-dependent vessels; the resulting hypoxia facilitates tumor stem-like cell transdifferentiation and VM.

Although VM can occur in a tumor microenvironment with high IFP, the space requirements for VM formation are unclear. Some networks of darkly stained tumor cells were observed when VM was initiated in a very small melanoma [53]. These networks invariably accompanied VM channels and were even connected to VM channels [53]. When Zhao and Han, et al. used a computer image analysis and a computer $3 \mathrm{D}$ rebuilding technique to analyze the relation of these cells and microvessels depended on tumor growth, they found that the peak value of these special cells appearance was followed by the peak value of VM channels formation in time line in a B16 melanoma-bearing mouse model [54] (unpublished data). The correlation between VM network and these cells in space and time suggests that these cells are pioneers in VM formation. Immunohistochemistry, transferase-mediated deoxyuridine triphosphate-biotin nick end labeling (TUNEL) staining and electron microscopy revealed that these cells exhibited a necrotic morphology, but expressed some apoptosis-associated proteins [53]. We coined the term "linearly patterned programmed cell necrosis" (LPPCN) to describe them [53]. LPPCN and VM coexistence predicts poor prognosis in human melanoma patients. Furthermore, high IFP and hypoxia were shown to promote LPPCN and VM channels. Tumor cells undergoing LPPCN under conditions of high IFP and hypoxia expressed increased Bax, Bcl-2, cytochrome c, caspase3, caspase9, calpain 1, caspase 8 and Fas. Inhibitors of caspase 3, caspase 8 and caspase 9 also inhibited LPPCN and VM (unpublished data).

In $\mathrm{HCC}$, the proapoptotic factor bcl-2 is positively correlated with VM and with nuclear expression of EMTregulating transcription factor Twist1 [55]. Hypoxia induces Bcl-2 and Twist1 coexpression in HCC HpG2 cell nuclei, and co-immunoprecipitation and knockdown experiments found that two separate Bcl-2 domains bound to a Twist1helix-loop-helix DNA binding domain [5556]. The Bcl-2/Twist1 complex facilitates Twist1 nuclear transport and leads to transcriptional activation of a wide range of genes that induce tumor cell EET and VM [55]. Hence, LPPCN not only provides the spatial basis for VM channels, but also promotes VM through the interaction of LPPCN-related proteins and EMT factors.

\section{ECM remodeling}

ECM remodeling is a critical step in VM channel formation and angiogenesis. A PAS-positive ECM layer coats the internal surfaces of VM channels. Known PASpositive substances include laminin, collagens IV and VI, mucopolysaccharide, HSPG and F-tissue factor (TF), TF pathway inhibitor 1 (TFPI-1) and 2 (TFPI-2) [2, 57]. Blood vessel basement membranes also include laminin, collagens IV and VI, and mucopolysaccharide [2]. Hence, ECM remodeling is needed for both VM channel construction and to facilitate connection of VM and endothelium-dependent vessels $[2,58]$. A balance of TF and TFPI-1 is thought to modulate anticoagulant functions to maintain flow in VM channels [57]. 
Matrix metalloproteases (MMPs) have important functions in ECM remodeling for VM channel formation. In melanoma, both MMP-14 and MMP-2 degrade laminin$5 \gamma 2$-chain into promigratory $\gamma 2^{\prime}$ and $\gamma 2 x$ fragments, which then stimulate invasion and VM formation [59]. MMP2 and MMP-9 are linked to VM and poor prognosis in cancers of the breast, ovaries, prostate, liver, stomach and kidneys, along with GISTs, mesothelial sarcomas, rhabdomyosarcomas and melanomas [6, 9-10, 13-14, 16]. Recently, ECM remodeling in VM was shown to be a chain reaction of EET. Overexpression of EMT transcription factor Twist1 under hypoxic conditions increased VE-cadherin, MMP-2 and MMP-9 expression and VM formation in HCC cells [10]. Twist1 is positively correlated with VE-cadherin, MMP-2, and MMP-9 expression in VM-positive human HCC [10]. VE-cadherin is an endothelium marker and a promoter of ECM remodeling in VM, and can phosphorylate erythropoietinproducing HCC-A2 (EphA2), activating PI3K to promote MMP-14 and MMP-2. Activated MMP-14 and MMP-2 degrade ECM to facilitate VM channel formation [24, 59].

Recently, Zhao,et al. found that collagenase-3 (MMP-13) specifically inhibits ECM remodeling in VM. Unlike MMP-2, -9 and -14, MMP-13 inhibits VM in melanoma. MMP-2 and MMP-14 degrade laminin-5 into 105-kDa laminin-5 $\gamma 2^{\prime}$ and 80-kDa laminin-5 $\gamma 2 \mathrm{x}$, which MMP-13 then cleaves into $\sim 40-\mathrm{kDa}$ fragments that are not used in VM [60]. MMP-13 also cleaves VE-cadherin to bind to $\beta$-catenin, forming a complex that inhibits $\beta$-catenin translocation, thus inhibiting VM and melanoma invasion [60].

\section{TRANSLATIONALSIGNIFICANCEOFEET AND CSCS IN VM}

In malignant tumors, VM is a marker for poor prognosis and presents a challenge to traditional antiangiogenic treatments. Most anti-angiogenic agents in clinics and in trials target endothelium-dependent vessels by blocking VEGF, VEFGRs, epidermal growth factor receptor (EGFR), or platelet-derived growth factor receptors (PDGFRs) [61]. These anti-angiogenic agents cannot inhibit tumor growth in many individual patients, and in some cases can promote tumor invasion and metastasis [61-62]. The precise reasons for treatment failure with these anti-angiogenic agents are not clear. The mechanisms of VM formation differ from those of endothelium-dependent vessels; VM channels also expose tumor cells directly to blood flow, which facilitates metastasis and may reduce the efficacy of conventional anti-angiogenic agents in VM-positive cases. For example, TNBCs that had been treated with sunitinib regrew and metastasized after sunitinib administration was terminated,

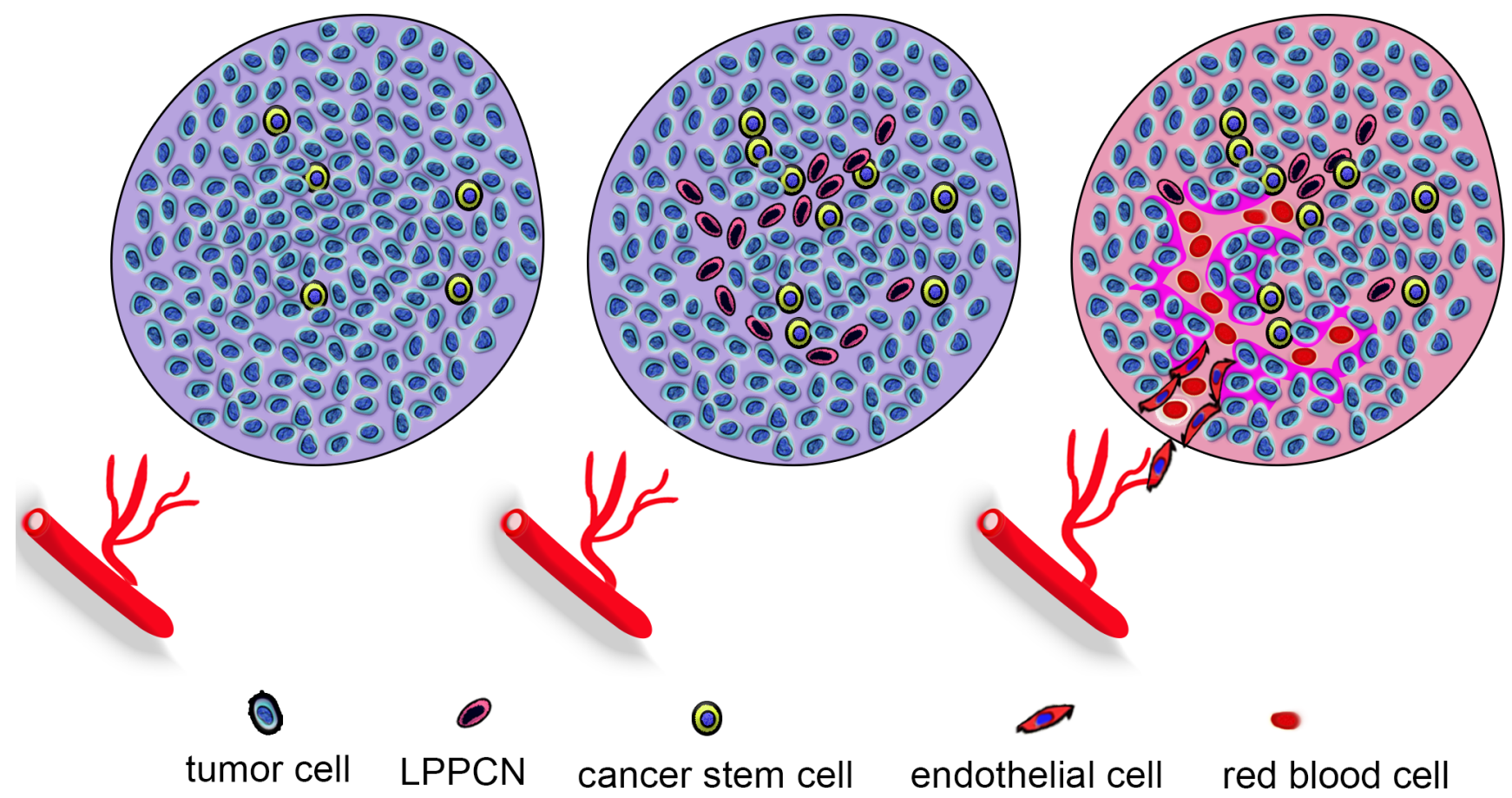

Figure 1: Hypoxia and IFP induce VM formation. When endothelium-dependent vessels do not grow into the tumor, the resulting hypoxia promotes VM formation to provide selective perfusion, leading to tumor aggression and metastasis. CSCs and EET accelerateVM formation by inducing tumor cell plasticity, remodeling ECM, and connecting VM channels and host blood vessels. Hypoxia and high IFP in the tumor microenvironment induce LPPCN in some tumor cells, to provide a spatial guide for VM and endothelium-dependent networks. EMT-inducing transcription factors may induce CSCs to differentiate into endothelium-like VE-cadherin+ cells in order to form VM channels with ECM remodeling. These channels then connect to endothelium-dependent vessels and areperfused withblood. 
as sunitinib treatment induced hypoxia, leading to formation of new VM channels [19]. After discontinuing treatment, endothelial vessels rebounded and linked to these VM channels [19]. The angiogenesis inhibitor, endostatin (an integrin signaling inhibitor), also failed to suppress VM channels formed by human melanoma MUM-2B and C8161 cells in vitro [21]. VM must be considered when devising strategies that utilize standard VEGF-targeted therapies or endothelium-dependent drugs.

Thalidomide is the first drug that has been proved to inhibit VM formation in mouse melanoma [63]. Thalidomide is a teratogenic sedative and a reactive oxygen species producer that targets TNF- $\alpha$. It can reduce expression of MMP-2 and MMP-9. In addition to blocking ECM remodeling, thalidomide also inhibits VM by interfering with anti-apoptotic, pro-angiogenesis and pro-metastatic functions of the NF- $\mathrm{KB}$ pathway [63]. Other drugs that have been shown to inhibit VM include the mTOR-specific inhibitor rapamycin, which reduced VM channels formed by malignant U87glioblastoma cells by inhibiting MMPs and HIF-1 $\alpha[64]$. Genistein and curcumin, both botanical extracts, can inhibit VE-cadherin $\rightarrow$ EphA2 $\rightarrow$ MMPs cascade to inhibit VM in melanoma in vivo [(70-72)]. A better understanding of VM channel formation will provide additional potential molecular targets for anti-angiogenic therapies.

As CSCs and EET have been shown to promote $\mathrm{VM}$ in malignant tumors, molecular regulators of EET and CSCs are candidate therapeutic targets in VMpositive tumors. Doxycycline, a tetracycline derivative, prolonged survival inmice with human liver tumors by inhibiting degradation of E-cadherin, thus preventing EET and VM. In vitro experiments implicated methylation of the E-cadherin gene and downregulation of the EMT promoters, vimentin and VE-cadherin, in the prevention of EET and VM [65].

The Notch pathway in CSCs is another possible target for VM inhibition. Anti-Notch4 antibody impaired VM in C8161 and SK-MEL-28 melanoma cells, and diminished clonogenicity in vitro by downregulating Nodal expression [34]. Similarly, shTwist1 disrupted VM in MDA-MB-231 TNBC cells grown on 3D matrigel under hypoxic conditions by reducing the CSC population[19]. Moreover, dual antiplatelet therapy, a gamma-secretase inhibitor, was found to inhibit glioblastoma CSCs from differentiating into endothelial-like progenitor cells through blockade of Dll4-Notch signaling [31]. A single treatment of bevacizumab, a recombinant humanized VEGF monoclonal antibody, did not influence the function of CSCs. However, the combination of both remarkably inhibited tumor growth in a mouse model with glioblastoma and fibrosarcoma engrafts [31]. These results suggest that a combination of anti-angiogenic therapies against multiple targets would be efficacious in malignant tumors with VM.

\section{CONCLUSIONS}

The functional tumor microcirculation system includes VM, endothelium-dependent vessels and mosaic vessels. When endothelium-dependent vessels are inhibited by traditional anti-angiogenic therapies, the resulting hypoxia promotes VM formation to provide selective perfusion, leading to tumor aggression and metastasis. CSCs and EET promote VM formation by accelerating tumor cell plasticity, remodeling ECM, and connecting VM channels and host blood vessels. Hypoxia and high IFP in the tumor microenvironment induce LPPCN in some tumor cells to provide a spatial guide for VM and endothelium-dependent networks. EMT-inducing transcription factors may induce CSCs to differentiate into endothelium-like VE-cadherin+ cells in order to form VM channels with ECM remodeling. These channels can then connect to endothelium-dependent vessels and be perfused with blood (Figure 1). The molecular regulators HIF-1 $\alpha$, Twist1, Notch4, VE-cadherin, EphA2, MMP-2, MMP-9 and MMP-13 are potential therapeutic targets and prognostic indicators.

\section{ACKNOWLEDGMENTS}

This study was supported by the Key Project of the National Nature Science Foundation of China (No. 81230050) and the Project of the National Nature Science Foundation of China (Nos. 81172046 and 81572872).

\section{CONFLICTS OF INTEREST}

The authors declare no conflicts of interest.

\section{REFERENCES}

1. Folkman J. Tumor angiogenesis: therapeutic implications. N Engl J Med. 1971; 285:1182-1186.

2. Maniotis AJ, Folberg R, Hess A, Seftor EA, Gardner LM, Pe'er J, Trent JM, Meltzer PS and Hendrix MJ. Vascular channel formation by human melanoma cells in vivo and in vitro: vasculogenic mimicry. Am J Pathol. 1999; 155:739752.

3. Zhang S, Zhang D and Sun B. Vasculogenic mimicry: current status and future prospects. Cancer Lett. 2007; 254:157-164.

4. Zhang S, Zhang D, Wang Y, Zhao W, Guo H, Zhao X and Sun B. Morphologic research of microcirculation patterns in human and animal melanoma. Med Oncol. 2006; 23:403409.

5. Sun BC, Zhang SW, Zhao XL and Hao XS. [Study on vasculogenic mimicry in malignant melanoma]. [Article in Chinese]. Zhonghua Bing Li Xue Za Zhi. 2003; 32:539-543.

6. Sun $\mathrm{B}$, Zhang $\mathrm{S}$, Zhao $\mathrm{X}$, Zhang $\mathrm{W}$ and Hao $\mathrm{X}$. Vasculogenic mimicry is associated with poor survival 
in patients with mesothelial sarcomas and alveolar rhabdomyosarcomas. Int J Oncol. 2004; 25:1609-1614.

7. Yao X, Ping Y, Liu Y, Chen K, Yoshimura T, Liu M, Gong W, Chen C, Niu Q, Guo D, Zhang X, Wang JM and Bian X. Vascular endothelial growth factor receptor 2 (VEGFR-2) plays a key role in vasculogenic mimicry formation, neovascularization and tumor initiation by Glioma stem-like cells. PLoS One. 2013; 8:e57188.

8. Liu TJ, Sun BC, Zhao XL, Zhao XM, Sun T, Gu Q, Yao Z, Dong XY, Zhao N and Liu N. CD133+ cells with cancer stem cell characteristics associates with vasculogenic mimicry in triple-negative breast cancer. Oncogene. 2013; 32:544-553.

9. Du J, Sun B, Zhao X, Gu Q, Dong X, Mo J, Sun T, Wang J, Sun R and Liu Y. Hypoxia promotes vasculogenic mimicry formation by inducing epithelial-mesenchymal transition in ovarian carcinoma. Gynecol Oncol. 2014; 133:575-583.

10. Sun T, Zhao N, Zhao XL, Gu Q, Zhang SW, Che N, Wang XH, Du J, Liu YX and Sun BC. Expression and functional significance of Twist1 in hepatocellular carcinoma: its role in vasculogenic mimicry. Hepatology. 2010; 51:545-556.

11. Li M, Gu Y, Zhang Z, Zhang S, Zhang D, Saleem AF, Zhao $\mathrm{X}$ and Sun B. Vasculogenic mimicry: a new prognostic sign of gastric adenocarcinoma. Pathol Oncol Res. 2010; 16:259-266.

12. Wang W, Lin $P$, Han C, Cai W, Zhao X and Sun B. Vasculogenic mimicry contributes to lymph node metastasis of laryngeal squamous cell carcinoma. J Exp Clin Cancer Res. 2010; 29:60.

13. Sun B, Qie S, Zhang S, Sun T, Zhao X, Gao S, Ni C, Wang X, Liu Y and Zhang L. Role and mechanism of vasculogenic mimicry in gastrointestinal stromal tumors. Hum Pathol. 2008; 39:444-451.

14. Hao X, Sun B, Zhang S and Zhao X. [Microarray study of vasculogenic mimicry in bi-directional differentiation malignant tumor]. [Article in Chinese]. Zhonghua Yi Xue Za Zhi. 2002; 82:1298-1302.

15. Scavelli C, Nico B, Cirulli T, Ria R, Di Pietro G, Mangieri D, Bacigalupo A, Mangialardi G, Coluccia AM, Caravita T, Molica S, Ribatti D, Dammacco F, et al. Vasculogenic mimicry by bone marrow macrophages in patients with multiple myeloma. Oncogene. 2008; 27:663-674.

16. Zhang Y, Sun B, Zhao X, Liu Z, Wang X, Yao X, Dong X and Chi J. Clinical significances and prognostic value of cancer stem-like cells markers and vasculogenic mimicry in renal cell carcinoma. J Surg Oncol. 2013; 108:414-419.

17. Wagenblast E, Soto M, Gutierrez-Angel S, Hartl CA, Gable AL, Maceli AR, Erard N, Williams AM, Kim SY, Dickopf S, Harrell JC, Smith AD, Perou CM, et al. A model of breast cancer heterogeneity reveals vascular mimicry as a driver of metastasis. Nature. 2015; 520:358-362.

18. Sun B, Zhang S, Zhang D, Du J, Guo H, Zhao X, Zhang W and Hao X. Vasculogenic mimicry is associated with high tumor grade, invasion and metastasis, and short survival in patients with hepatocellular carcinoma. Oncol Rep. 2006; 16:693-698.

19. Zhang D, Sun B, Zhao X, Ma Y, Ji R, Gu Q, Dong X, Li J, Liu F, Jia X, Leng X, Zhang C, Sun R, et al. Twist1 expression induced by sunitinib accelerates tumor cell vasculogenic mimicry by increasing the population of CD133+ cells in triple-negative breast cancer. Mol Cancer. 2014; 13:207.

20. Yao L, Sun B, Zhao X, Gu Q, Dong X, Zheng Y, Sun J, Cheng R, Qi H and An J. Overexpression of Wnt5a promotes angiogenesis in NSCLC. Biomed Res Int. 2014; 2014:832562.

21. van der Schaft DW, Seftor RE, Seftor EA, Hess AR, Gruman LM, Kirschmann DA, Yokoyama Y, Griffioen AW and Hendrix MJ. Effects of angiogenesis inhibitors on vascular network formation by human endothelial and melanoma cells. J Natl Cancer Inst. 2004; 96:1473-1477.

22. Hendrix MJ, Seftor EA, Hess AR and Seftor RE. Molecular plasticity of human melanoma cells. Oncogene. 2003; 22:3070-3075.

23. Hendrix MJ, Seftor EA, Kirschmann DA, Quaranta V and Seftor RE. Remodeling of the microenvironment by aggressive melanoma tumor cells. Ann N Y Acad Sci. 2003; 995:151-161.

24. Hess AR, Margaryan NV, Seftor EA and Hendrix MJ. Deciphering the signaling events that promote melanoma tumor cell vasculogenic mimicry and their link to embryonic vasculogenesis: role of the Eph receptors. Dev Dyn. 2007; 236:3283-3296.

25. Semenza GL. Vasculogenesis, angiogenesis, and arteriogenesis: mechanisms of blood vessel formation and remodeling. J Cell Biochem. 2007; 102:840-847.

26. Seftor EA, Meltzer PS, Schatteman GC, Gruman LM, Hess AR, Kirschmann DA, Seftor RE and Hendrix MJ. Expression of multiple molecular phenotypes by aggressive melanoma tumor cells: role in vasculogenic mimicry. Crit Rev Oncol Hematol. 2002; 44:17-27.

27. Bittner M, Meltzer P, Chen Y, Jiang Y, Seftor E, Hendrix M, Radmacher M, Simon R, Yakhini Z, Ben-Dor A, Sampas N, Dougherty E, Wang E, et al. Molecular classification of cutaneous malignant melanoma by gene expression profiling. Nature. 2000; 406:536-540.

28. Lehmann BD, Bauer JA, Chen X, Sanders ME, Chakravarthy AB, Shyr Y and Pietenpol JA. Identification of human triple-negative breast cancer subtypes and preclinical models for selection of targeted therapies. J Clin Invest. 2011; 121:2750-2767.

29. Sun D, Sun B, Liu T, Zhao X, Che N, Gu Q, Dong X, Yao Z, Li R, Li J, Chi J and Sun R. Slug promoted vasculogenic mimicry in hepatocellular carcinoma. J Cell Mol Med. 2013; 17:1038-1047.

30. Francescone R, Scully S, Bentley B, Yan W, Taylor SL, Oh D, Moral L and Shao R. Glioblastoma-derived tumor cells induce vasculogenic mimicry through Flk-1 protein 
activation. J Biol Chem. 2012; 287:24821-24831.

31. Wang R, Chadalavada K, Wilshire J, Kowalik U, Hovinga KE, Geber A, Fligelman B, Leversha M, Brennan C and Tabar V. Glioblastoma stem-like cells give rise to tumour endothelium. Nature. 2010; 468:829-833.

32. Ricci-Vitiani L, Pallini R, Biffoni M, Todaro M, Invernici G, Cenci T, Maira G, Parati EA, Stassi G, Larocca LM and De Maria R. Tumour vascularization via endothelial differentiation of glioblastoma stem-like cells. Nature. 2010; 468:824-828.

33. Rangel MC, Karasawa H, Castro NP, Nagaoka T, Salomon DS and Bianco C. Role of Cripto-1 during epithelial-tomesenchymal transition in development and cancer. Am J Pathol. 2012; 180:2188-2200.

34. Hardy KM, Kirschmann DA, Seftor EA, Margaryan NV, Postovit LM, Strizzi L and Hendrix MJ. Regulation of the embryonic morphogen Nodal by Notch4 facilitates manifestation of the aggressive melanoma phenotype. Cancer Res. 2010; 70:10340-10350.

35. Drasin DJ, Robin TP and Ford HL. Breast cancer epithelialto-mesenchymal transition: examining the functional consequences of plasticity. Breast Cancer Res. 2011; 13:226.

36. Zeisberg $\mathrm{M}$ and Neilson EG. Biomarkers for epithelialmesenchymal transitions. J Clin Invest. 2009; 119:14291437.

37. McCoy EL, Iwanaga R, Jedlicka P, Abbey NS, Chodosh LA, Heichman KA, Welm AL and Ford HL. Six1 expands the mouse mammary epithelial stem/progenitor cell pool and induces mammary tumors that undergo epithelialmesenchymal transition. J Clin Invest. 2009; 119:26632677.

38. Che N, Zhao XL, Sun T, Zhao XM, Gu Q, Dong XY, Zhao N, Liu YR, Yao Z and Sun BC. The role of Twist1 in hepatocellular carcinoma angiogenesis: a clinical study. Hum Pathol. 2011; 42:840-847.

39. Liu Z, Sun B, Qi L, Li H, Gao J and Leng X. Zinc finger E-box binding homeobox 1 promotes vasculogenic mimicry in colorectal cancer through induction of epithelial-tomesenchymal transition. Cancer Sci. 2012; 103:813-820.

40. Qi L, Sun B, Liu Z, Li H, Gao J and Leng X. Dickkopf-1 inhibits epithelial-mesenchymal transition of colon cancer cells and contributes to colon cancer suppression. Cancer Sci. 2012; 103:828-835.

41. Cheng R, Sun B, Liu Z, Zhao X, Qi L, Li Y and Gu Q. Wnt5a suppresses colon cancer by inhibiting cell proliferation and epithelial-mesenchymal transition. J Cell Physiol. 2014; 229:1908-1917.

42. Lee SL, Rouhi P, Dahl Jensen L, Zhang D, Ji H, Hauptmann G, Ingham P and Cao Y. Hypoxia-induced pathological angiogenesis mediates tumor cell dissemination, invasion, and metastasis in a zebrafish tumor model. Proc Natl Acad Sci U S A. 2009; 106:19485-19490.

43. Notte A, Ninane N, Arnould T and Michiels C. Hypoxia counteracts taxol-induced apoptosis in MDA-MB-231 breast cancer cells: role of autophagy and JNK activation. Cell Death Dis. 2013; 4:e638.

44. Shams I, Avivi A and Nevo E. Hypoxic stress tolerance of the blind subterranean mole rat: expression of erythropoietin and hypoxia-inducible factor 1 alpha. Proc Natl Acad Sci U S A. 2004; 101:9698-9703.

45. Compernolle V, Brusselmans K, Acker T, Hoet P, Tjwa M, Beck H, Plaisance S, Dor Y, Keshet E, Lupu F, Nemery B, Dewerchin M, Van Veldhoven P, et al. Loss of HIF2alpha and inhibition of VEGF impair fetal lung maturation, whereas treatment with VEGF prevents fatal respiratory distress in premature mice. Nat Med. 2002; 8:702-710.

46. Olenyuk BZ, Zhang GJ, Klco JM, Nickols NG, Kaelin WG, Jr. and Dervan PB. Inhibition of vascular endothelial growth factor with a sequence-specific hypoxia response element antagonist. Proc Natl Acad Sci U S A. 2004; 101:1676816773.

47. Sun B, Zhang D, Zhang S, Zhang W, Guo H and Zhao $X$. Hypoxia influences vasculogenic mimicry channel formation and tumor invasion-related protein expression in melanoma. Cancer Lett. 2007; 249:188-197.

48. Mao XG, Xue XY, Wang L, Zhang X, Yan M, Tu YY, Lin W, Jiang XF, Ren HG, Zhang W and Song SJ. CDH5 is specifically activated in glioblastoma stemlike cells and contributes to vasculogenic mimicry induced by hypoxia. Neuro Oncol. 2013; 15:865-879.

49. van der Schaft DW, Hillen F, Pauwels P, Kirschmann DA, Castermans K, Egbrink MG, Tran MG, Sciot R, Hauben E, Hogendoorn PC, Delattre O, Maxwell PH, Hendrix MJ, et al. Tumor cell plasticity in Ewing sarcoma, an alternative circulatory system stimulated by hypoxia. Cancer Res. 2005; 65:11520-11528.

50. Wang Z, Dabrosin C, Yin X, Fuster MM, Arreola A, Rathmell WK, Generali D, Nagaraju GP, El-Rayes B, Ribatti D, Chen YC, Honoki K, Fujii H, et al. Broad targeting of angiogenesis for cancer prevention and therapy. Semin Cancer Biol. 2015; 35 Suppl:S224-43. doi: 10.1016/j.semcancer.2015.01.001.

51. Sun B, Zhang S, Zhang D, Gu Y, Zhang W and Zhao X. The influence of different microenvironments on melanoma invasiveness and microcirculation patterns: an animal experiment study in the mouse model. J Cancer Res Clin Oncol. 2007; 133:979-985.

52. Joensuu H. Gastrointestinal stromal tumor (GIST). Ann Oncol. 2006; 17 Suppl 10:x280-286.

53. Zhang S, Li M, Zhang D, Xu S, Wang X, Liu Z, Zhao X and Sun B. Hypoxia influences linearly patterned programmed cell necrosis and tumor blood supply patterns formation in melanoma. Lab Invest. 2009; 89:575-586.

54. Han C, Sun BU, Wang W, Cai WJ, Lou D, Sun Y and Zhao X. A pilot study on morphology and the mechanism involved in linearly patterned programmed cell necrosis in melanoma. Oncol Lett. 2010; 1:821-826. 
55. Sun T, Sun BC, Zhao XL, Zhao N, Dong XY, Che N, Yao Z, Ma YM, Gu Q, Zong WK and Liu ZY. Promotion of tumor cell metastasis and vasculogenic mimicry by way of transcription coactivation by Bcl-2 and Twist1: a study of hepatocellular carcinoma. Hepatology. 2011; 54:1690-1706.

56. Zhao N, Sun BC, Sun T, Ma YM, Zhao XL, Liu ZY, Dong $\mathrm{XY}$, Che N, Mo J and Gu Q. Hypoxia-induced vasculogenic mimicry formation via VE-cadherin regulation by Bcl-2. Med Oncol. 2012; 29:3599-3607.

57. Ruf W, Seftor EA, Petrovan RJ, Weiss RM, Gruman LM, Margaryan NV, Seftor RE, Miyagi Y and Hendrix MJ. Differential role of tissue factor pathway inhibitors 1 and 2 in melanoma vasculogenic mimicry. Cancer Res. 2003; 63:5381-5389.

58. Hao XS, Sun BC, Zhang SW and Zhao XL. [Correlation between the expression of collgen IV, VEGF and vasculogenic mimicry]. [Article in Chinese]. Zhonghua Zhong Liu Za Zhi. 2003; 25:524-526.

59. Seftor RE, Seftor EA, Koshikawa N, Meltzer PS, Gardner LM, Bilban M, Stetler-Stevenson WG, Quaranta V and Hendrix MJ. Cooperative interactions of laminin 5 gamma2 chain, matrix metalloproteinase-2, and membrane type1-matrix/metalloproteinase are required for mimicry of embryonic vasculogenesis by aggressive melanoma. Cancer Res. 2001; 61:6322-6327.

60. Zhao X, Sun B, Li Y, Liu Y, Zhang D, Wang X, Gu Q, Zhao J, Dong X, Liu Z and Che N. Dual effects of collagenase-3 on melanoma: metastasis promotion and disruption of vasculogenic mimicry. Oncotarget. 2015; 6:8890-9. doi: 10.18632/oncotarget.3189.
61. Cao Y, Arbiser J, D'Amato RJ, D'Amore PA, Ingber DE, Kerbel R, Klagsbrun M, Lim S, Moses MA, Zetter B, Dvorak $\mathrm{H}$ and Langer R. Forty-year journey of angiogenesis translational research. Sci Transl Med. 2011; 3:114rv113.

62. Paez-Ribes M, Allen E, Hudock J, Takeda T, Okuyama H, Vinals F, Inoue M, Bergers G, Hanahan D and Casanovas O. Antiangiogenic therapy elicits malignant progression of tumors to increased local invasion and distant metastasis. Cancer Cell. 2009; 15:220-231.

63. Zhang S, Li M, Gu Y, Liu Z, Xu S, Cui Y and Sun B. Thalidomide influences growth and vasculogenic mimicry channel formation in melanoma. J Exp Clin Cancer Res. 2008; 27:60.

64. Huang M, Ke Y, Sun X, Yu L, Yang Z, Zhang Y, Du M, Wang J, Liu X and Huang S. Mammalian target of rapamycin signaling is involved in the vasculogenic mimicry of glioma via hypoxia-inducible factor-1alpha. Oncol Rep. 2014; 32:1973-1980.

65. Meng J, Sun B, Zhao X, Zhang D, Gu Q, Dong X, Zhao $\mathrm{N}$, Liu P and Liu Y. Doxycycline as an inhibitor of the epithelial-to-mesenchymal transition and vasculogenic mimicry in hepatocellular carcinoma. Mol Cancer Ther. 2014; 13:3107-3122. 